\title{
Oxygen Uptake Kinetics: Implications for Endurance Running Success
}

\author{
Richard L. Hughson and Azmy Faisal
}

The kinetics of oxygen uptake $\left(\dot{V} \mathrm{O}_{2}\right)$ reflect the rate at which the oxidative energy supply system is adapting to the demands of a new, higher, work rate. An athlete starting a 5,000 $\mathrm{m}$ race requires rapid adaptation of oxidative phosphorylation to minimize the contribution of phosphocreatine and anaerobic glycolysis with lactate accumulation during the period of oxygen deficit. This will enable the athlete to maintain reserves for the sprint to the finish. $\dot{V} \mathrm{O}_{2}$ kinetics are affected by both the rate at which metabolic pathways are activated and the ability to transport $\mathrm{O}_{2}$ to the exercising muscles. Following the onset of moderate intensity exercise, $\dot{V} \mathrm{O}_{2}$ increases in a fit athlete to attain a steady state within the first two minutes. However, for higher intensities of exercise such as 5,000 m running where the metabolic demand is approaching $\dot{V} O_{2 \max }$ the attainment of a steady state is delayed. In the case of the high intensity exercise, there is a corresponding slower response of cardiac output $(\dot{Q})$. Prior warm-up exercise at either moderate or heavy intensity has been shown recently to cause a more rapid increase in $\dot{Q}$ which then facilitates a more rapid increase in $\dot{V} \mathrm{O}_{2}$. This review highlights some of the recent evidence concerning the kinetics of $\dot{V} \mathrm{O}_{2}$ and $\dot{Q}$ in fit, endurance-trained, athletes during moderate and heavy intensity exercise.

\section{Introduction}

$\mathbf{F}$ or an elite endurance runner, the rate at which the oxidative supply of energy increases after the start of a race will contribute to competitive success. The ability to achieve the optimal balance of the energy supply systems as quickly as possible when racing will impact the rate and extent of glycogen depletion, the accumulation of lactate, and the depletion of high energy phosphocreatine (PCr) stores. The increase in oxidative energy supply can be characterized by the kinetics of oxygen uptake $\left(\dot{V} \mathrm{O}_{2}\right)$. For light to moderate intensities of exercise, $\dot{V} \mathrm{O}_{2}$ increases with a time course that can be adequately described by a simple exponential relationship with a single primary time constant (tau).

Richard L. Hughson Faculty of Applied Health Sciences, University of Waterloo, Waterloo, Ontario, Canada, Azmy Faisal Faculty of Physical Education for Men, Alexandria University, Alexandria, Egypt and Faculty of Applied Health Sciences, University of Waterloo, Waterloo, Ontario, Canada.
However, for heavier exercise such as that in racing $5,000 \mathrm{~m}$, the $\dot{V} \mathrm{O}_{2}$ increases in a more complex manner that often involves an additional slow component response that delays the athlete's ability to supply energy through oxidative metabolism. The time course of the primary response is very important to racing success as the slower kinetics are associated with greater accumulation of lactic acid that can result in fatigue. In this brief review, we will examine the basic concepts of $\dot{V} O_{2}$ kinetics for moderate and heavy intensity exercise, and we will describe how prior warm-up exercise impacts $\dot{\mathrm{V}} \mathrm{O}_{2}$ kinetics.

\section{$\dot{V} \mathrm{O}_{2}$ kinetics in moderate exercise}

Moderate intensity exercise requires a work rate below the intensity at the ventilatory threshold, or less than $60-70 \% \dot{V} O_{2 \max }$. Following the onset of moderate exercise $\dot{\mathrm{V}} \mathrm{O}_{2}$ increases in what seems to be well described as an exponential relationship (Figure 1). 

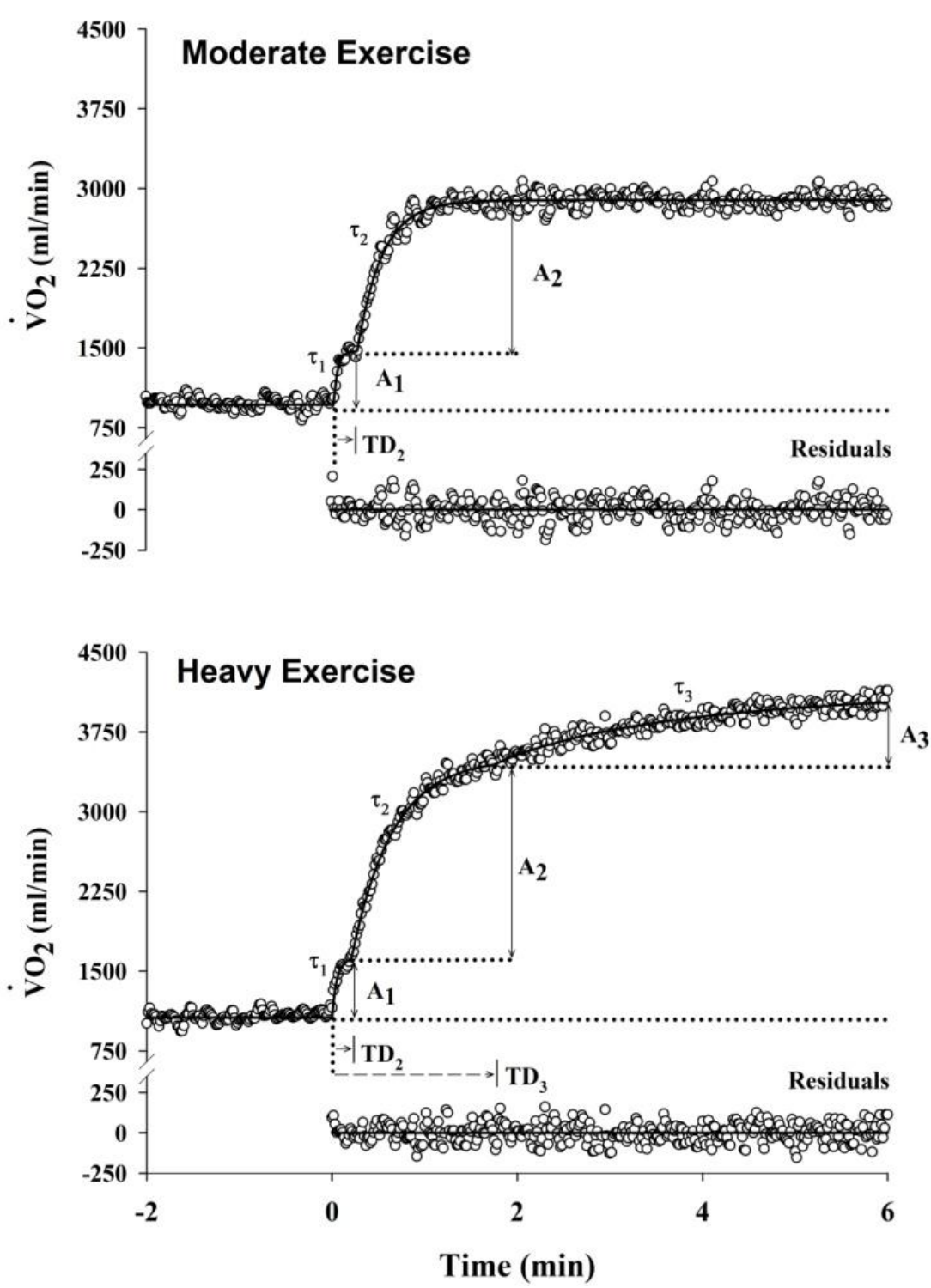

Figure 1: A typical response of $\dot{V} O_{2}$ is shown for an individual exercising at moderate (approximately $65 \% \dot{V} O_{2 \max }$, top panel) and heavy (approximately $85 \% \dot{V} O_{2 \max }$, bottom panel) intensity. The data points are the average of multiple repetitions by this individual determined for each 1s interval. The line of best fit shows a two component exponential response for moderate exercise and a three component exponential response for heavy exercise. The time course of the primary phase is described by a time constant which indicates the time required to reach $63 \%$ of the amplitude A2. The excess $\mathrm{O} 2$ consumption during heavy exercise (slow component) is represented by the amplitude A3. For more details about curve fitting (see (Faisal et al. 2009)).

Throughout the transition phase until steady state $\dot{V} \mathrm{O}_{2}$ is reached, energy is also supplied during moderate intensity efforts by $\mathrm{PCr}$ breakdown and possibly anaerobic glycolysis with some net accumulation of lactate (Figure 2 ). The contribution by these anaerobic energy sources is referred to as the "oxygen deficit" (Hohwu Christensen \& Hogberg, 1950). 


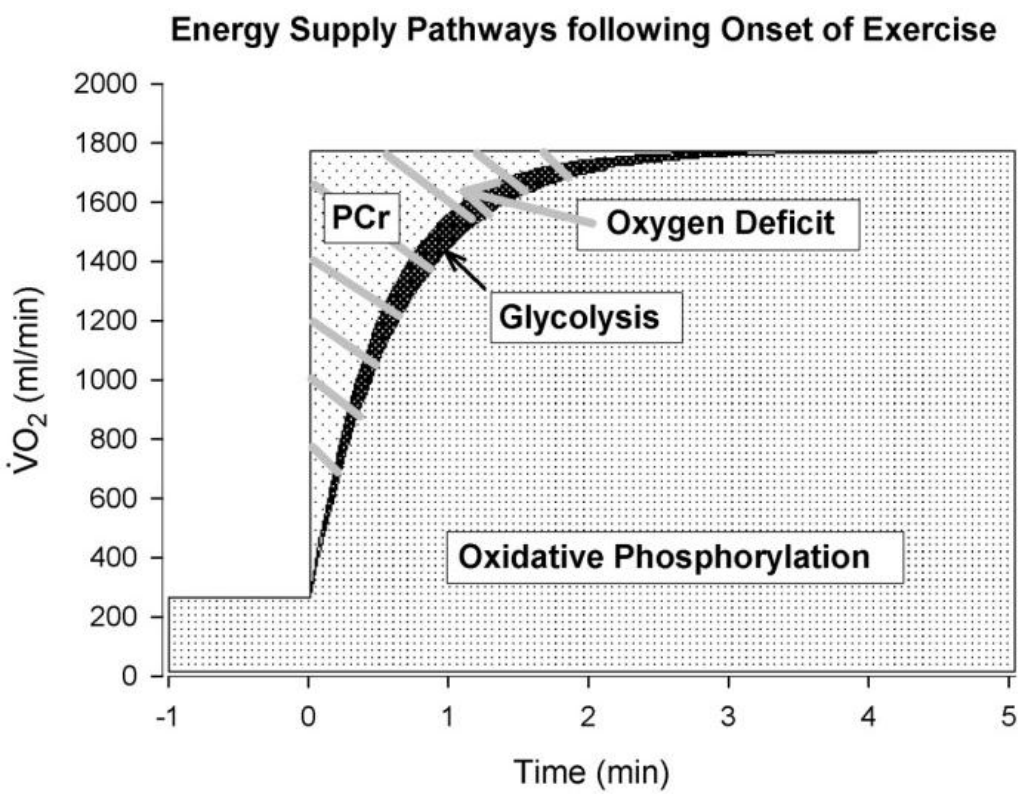

Figure 2: A schematic diagram of the relative contributions of the different energy supply pathways following the onset of moderate intensity exercise at approximately $65 \% \quad \dot{V} O_{2 \max }$. The metabolic energy requirement increased immediately with the start of exercise and initially phosphocreatine (PCr) stores of high energy phosphates contribute a major share to the total energy supply. Progressively oxidative phosphorylation increases and anaerobic glycolysis with lactate accumulation might provide some energy. The summation of the $\mathrm{PCr}$ and anaerobic glycolysis contributions comprise the oxygen deficit. Adapted from Hughson et al. (2001).

The rate at which $\dot{V} O_{2}$ increases following the onset of moderate intensity exercise is determined by the interaction of metabolic requirements and $\mathrm{O}_{2}$ supply. The coincidence between breakdown of $\mathrm{PCr}$ and $\dot{\mathrm{V}} \mathrm{O}_{2}$ kinetics has been described from time course investigations utilizing magnetic resonance spectroscopy (Rossiter et al., 1999). Some researchers have argued that this coincidence is evidence that $\dot{V} O_{2}$ kinetics during moderate intensity exercise are determined solely by the production of metabolic substrates in a so-called "metabolic inertia" (Greenhaff et al., 2002; Pringle et al., 2003). However, several lines of evidence suggest that the $\mathrm{O}_{2}$ transport mechanisms link closely with $\mathrm{O}_{2}$ utilization mechanisms to set the time course of $\dot{V} O_{2}$ kinetics.

When moderate intensity exercise was performed in a hypo- or hyperbaric environment to manipulate the inspired partial pressure of $\mathrm{O}_{2}$, there were direct links between $\mathrm{O}_{2}$ transport and the magnitude of the oxygen deficit. Linnarsson and colleagues (1974) investigated exercise that required $53 \%$ of sea level $\dot{V} \mathrm{O}_{2}$ under conditions of reduced barometric pressure $(0.68$ atmospheres) and elevated barometric pressure (1.4 atmospheres). Under these altered conditions, the exercise intensity was $62 \%$ and $47 \%$ of the corresponding $\dot{V} O_{2 \max }$ and would thus still be described as moderate. With reduced inspired $\mathrm{O}_{2}$, the magnitude of the oxygen deficit increased coincident with greater $\mathrm{PCr}$ depletion and higher muscle and blood lactate concentrations. Conversely with elevated inspired $\mathrm{O}_{2}$, the oxygen deficit was reduced, there was less depletion of $\mathrm{PCr}$ and muscle and blood lactate concentrations were lower than the sea level values.

The $\mathrm{O}_{2}$ transport at the onset of moderate exercise can also be manipulated by changes in the cardiac response. A slower increase in heart rate slowed the $\dot{V} O_{2}$ kinetics (Hughson \& Morrissey, 1982). Conversely, Faisal and colleagues (2009) recently demonstrated that elevated cardiac output ( $\dot{Q})$ as a consequence of prior heavy exercise was associated with an 
acceleration of $\dot{V} O_{2}$ kinetics. Indeed, these investigators found that the kinetics of $\dot{Q}$ had a similar time constant as $\dot{V} O_{2}$ during moderate exercise (Figure 3) and they observed for the first time that cardiac stroke volume had a transient overshoot in the early phase of exercise (Faisal et al., 2009).

$\dot{V} \mathrm{O}_{2}$ kinetics in heavy to severe exercise

The $\dot{V} O_{2}$ kinetics during heavy exercise have a phase of rapid adaptation in the primary component, but this component is not sufficient to meet the metabolic requirements of the exercise task and there is a further rise in $\dot{V} O_{2}$. When the work rate is selected to be approximately $50 \%$ of the difference between the work rate at the ventilatory threshold and $\dot{V} O_{2 \max }$, the $\dot{V} O_{2}$ will normally increase during this slower adaptive phase to an apparent plateau (Figure 1 bottom). The mechanisms that underlie the slower phase of $\dot{V} O_{2}$ kinetics are probably related to two distinct factors. First, the $\dot{V} O_{2}$ achieved at the end of the primary phase is insufficient to meet the requirements of oxidative phosphorylation. Second, because some muscle fibres relied upon increased anaerobic glycolysis during the oxygen deficit phase there was probably sufficient accumulation of lactate and hydrogen ions to induce muscle fatigue. Therefore, additional muscle fibres would have been recruited to accomplish the external work of the task.

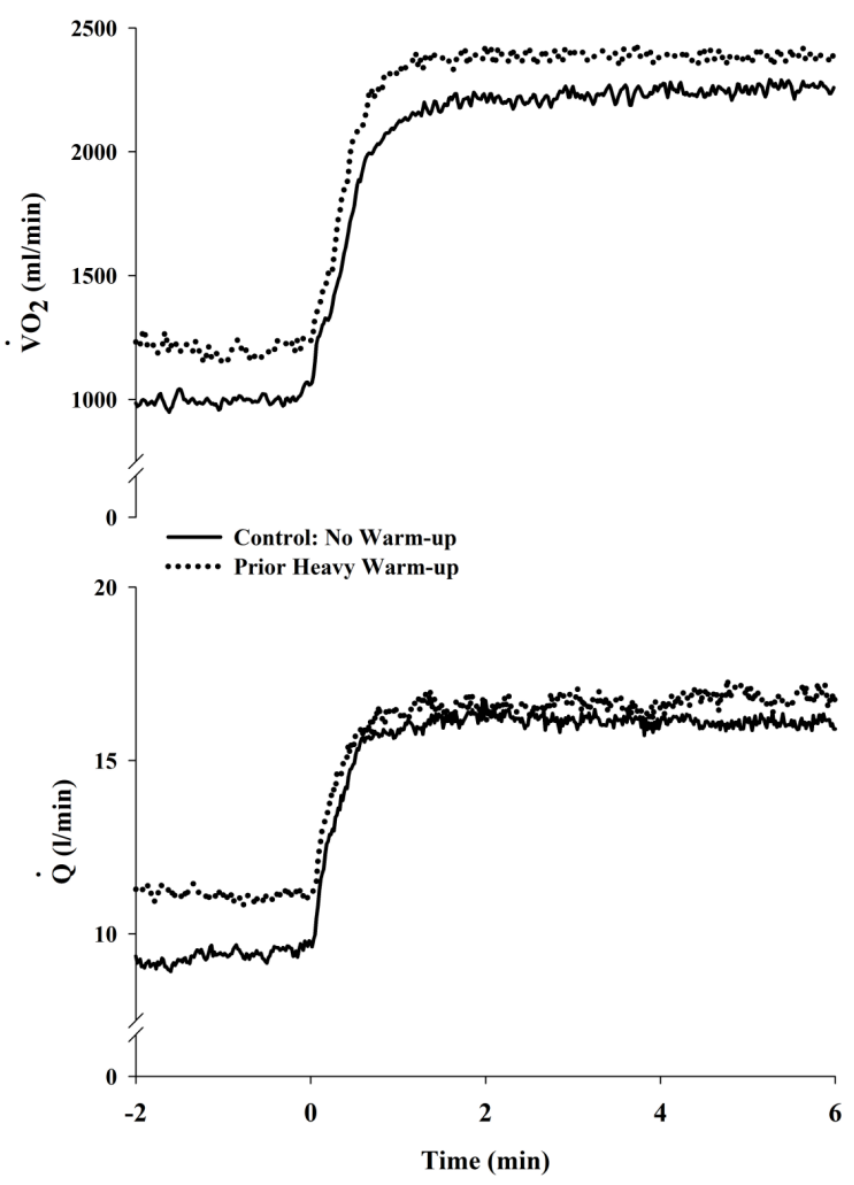

Figure 3: $\dot{V} \mathrm{O}_{2}$ (top) and $\dot{Q}$ (bottom) time series data for moderate cycling exercise. Data lines are the average of 9 subjects with $\geq 4$ repetitions per each exercise condition. (Solid lines, control; dotted lines, following prior heavy warm-up). Prior heavy exercise resulted in the acceleration of primary phase time constant (tau 2, see text for numbers) for both $\dot{V} O_{2}$ and $\dot{Q}$. Modified from Faisal et al. (2009). 
This latter mechanism probably becomes more important as the work rate increases to the severe intensity where the energy requirements exceed $\dot{V} O_{2 \max }$ and the $\dot{V} O_{2}$ during the exercise will never meet the metabolic requirements.

Kinetics of $\dot{V} \mathrm{O}_{2}$ during heavy exercise have been extensively studied, but the analyses of these results are subject to error (see (Hughson, 2009)). Several papers have reported that the time constant for the primary component of $\dot{V} O_{2}$ is unchanged or even faster than that observed for moderate intensity exercise (e.g. (Brittain et al., 2001; Pringle et al., 2003; Ozyener et al., 2001; Scheuermann et al., 2001)). However, in each of these cases the amplitude of the primary component was less than predicted. It is well established that over a wide range of work rates that the metabolic cost for cycle ergometer exercise is about $10 \mathrm{ml} / \mathrm{min} / \mathrm{W}$, so when the amplitude is reduced, it means that the mathematical model used to fit the data has been constrained to non-physiological parameter values. This invalidates the estimates for the time constants. The clearest examples of this can be seen with severe exercise. Hughson and colleagues (2000) used standard exponential curve fitting procedures to provide an estimate of the time constants for exercise at $57 \%, 96 \%$ and $125 \% \quad \dot{V} O_{2 \max }$. The time constant for the primary component was progressively reduced with the increasing work rate (Hughson et al., 2000). However, when the data were fitted accounting for the magnitude of the error signal induced by the power requirement, the estimates for the time constants were more than twice as long revealing slower kinetics. These data are consistent with the observation of Faisal et al. (2009) that the primary phase time constants for $\dot{V} \mathrm{O}_{2}$ and $\dot{Q}$ were slower during exercise at $85 \%$ $\dot{V} O_{2 \max }$ compared to exercise at $80 \%$ of ventilatory threshold.
Further, when the work rate metabolic requirement is increased above $\dot{V} O_{2 \max }$ the kinetics of $\dot{V} \mathrm{O}_{2}$ and $\dot{Q}$ would be expected to be further slowed (Hughson \& Faisal, 2010). These data collectively provide evidence that $\dot{V} O_{2}$ kinetics during heavy to severe exercise are regulated in large part by the kinetics of bulk $\mathrm{O}_{2}$ transport through $\dot{Q}$.

\section{Effect of prior warm-up exercise}

A detailed investigation of the effects of prior warm-up exercise has recently been published (Faisal et al., 2009). This study was the first to examine the integrated kinetics of $\dot{V} O_{2}$ and $\dot{Q}$ during transitions to moderate and heavy exercise with and without prior warm-up. Consistent with several (Gerbino et al., 1996; Tordi et al., 2003) but not all (Burnley et al., 2000) previous studies, it was observed that $\dot{\mathrm{V}} \mathrm{O}_{2}$ kinetics were accelerated when heavy exercise followed prior heavy exercise. A new finding from the study of Faisal et al. (2009) was that the acceleration of $\dot{V} O_{2}$ kinetics were accompanied by similar changes in $\dot{Q}$ kinetics (Figure 4). Indeed the time constant for $\dot{V} O_{2}$ improved $14 \%$ from $26.1 \pm 3.2 \mathrm{~s}$, when exercise was performed at $85 \%$ of $\dot{V} O_{2 \max }$ without a prior warm-up, to $22.1 \pm 2.9 \mathrm{~s}$ after a single bout of heavy prior exercise, and in the same exercise bouts, the kinetics of $\dot{Q}$ were faster by $19 \%$ from $27.4 \pm 7.2 \mathrm{~s}$ to $23.8 \pm 5.6 \mathrm{~s}$. Important new information about the ability of prior moderate exercise to accelerate the kinetics of $\dot{Q}$ and $\dot{V} O_{2}$ were also found in this study. After a moderate intensity warm-up the time constant for $\dot{Q}$ $(22.0 \pm 4.1 \mathrm{~s})$ as well as $\dot{\mathrm{V}} \mathrm{O}_{2}(22.5 \pm 2.7 \mathrm{~s})$ were faster than after no warm-up and almost identical to the acceleration noted after the heavy warm-up. 


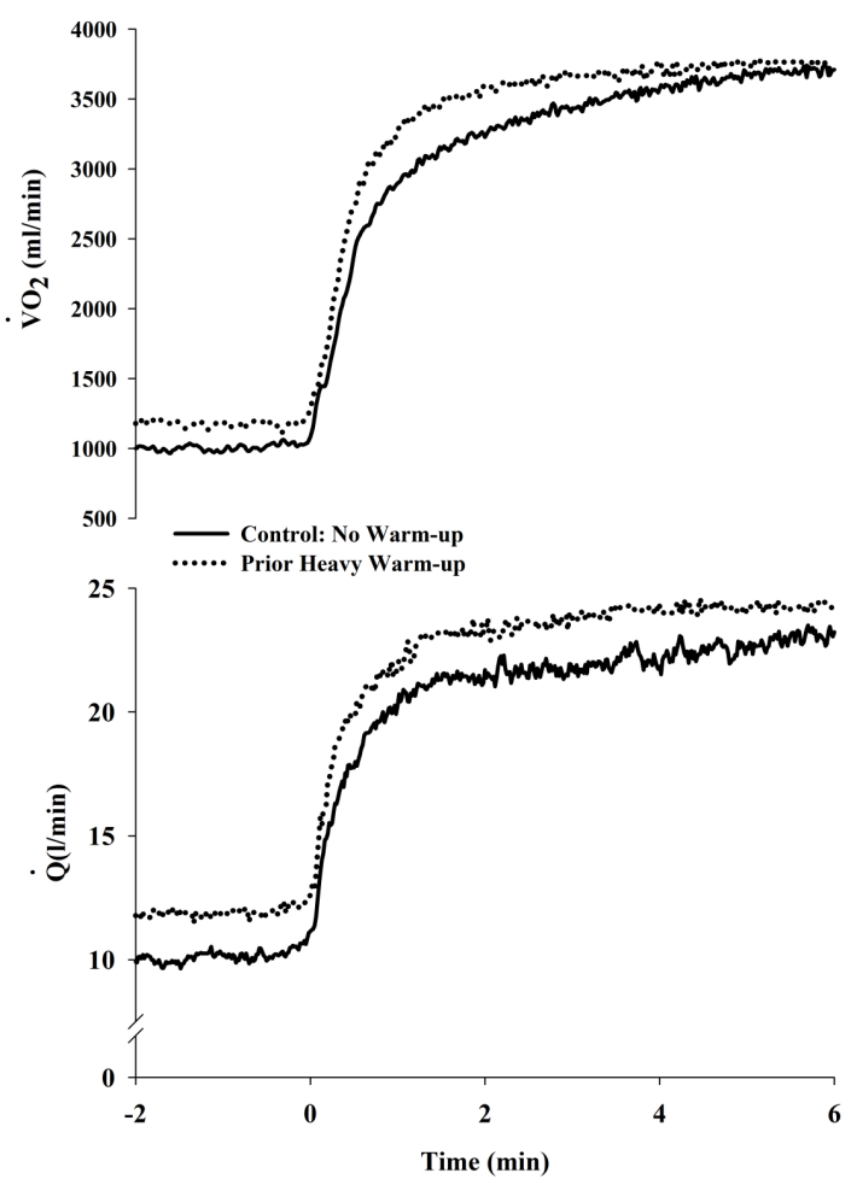

Figure 4: $\dot{V} \mathrm{O}_{2}$ (top) and $\dot{Q}$ (bottom) time series data for heavy cycling exercise. Data lines are the average of 9 subjects with $\geq 4$ repetitions per each exercise condition. (Solid lines, control; dotted lines, following prior heavy warm-up). Prior heavy exercise resulted in the acceleration of primary phase time constant (tau 2, see text for numbers) for both $\dot{V} O_{2}$ and $\dot{Q}$. Modified from Faisal et al. (2009).

Although the prior moderate and heavy exercise warm-ups appeared to have similar effects on the time constants for $\dot{V} O_{2}$ and $\dot{Q}$ during subsequent heavy exercise, the absolute $\dot{V} \mathrm{O}_{2}$ and $\dot{Q}$ were influenced differently. After a moderate intensity warm-up the $\dot{V} O_{2}$ and $\dot{Q}$ returned to their baseline values, but after the heavy warm-up the $\dot{V} O_{2}$ and $\dot{Q}$ were both significantly elevated (Figure 4). During the following bout of heavy exercise the prior baseline did impact the absolute values of $\dot{V} \mathrm{O}_{2}$ and $\dot{Q}$. For the moderate warm-up where baseline $\dot{V} O_{2}$ and $\dot{Q}$ were not different from the control baseline, $\dot{V} O_{2}$ and $\dot{Q}$ were elevated during the transition phase (as a consequence of the faster time constant) but they each reached similar plateau values to those attained during the control condition that did not have a prior warm-up. However, during exercise that followed the heavy prior warm-up both $\dot{V} O_{2}$ and $\dot{Q}$ were significantly elevated until near the completion of the 5-minute exercise bout (Figure 4). Factors affected by the heavy warmup related to the thermoregulatory and metabolic load contributed to the increase in $\dot{Q}$. The higher $\dot{V} \mathrm{O}_{2}$ might have been a consequence of the need to recruit additional muscle fibres if some fatigue had been caused by the prior heavy warm-up.

The impact of prior warm-up on the metabolic state was also evident from the blood lactate concentration in the study of Faisal et al. (2009). When there was no prior exercise the baseline lactate concentration was $0.86 \pm 0.18 \mathrm{mM}$, and after a moderate intensity warm-up blood lactate 
concentration was still low at $1.07 \pm 0.21 \mathrm{mM}$. However when a prior heavy exercise bout was used as the warm-up blood lactate had increased to $4.93 \pm 0.81 \mathrm{mM}$. The consequence of the elevated lactate concentration after a heavy exercise warm-up was that at the end of a heavy exercise bout lactate was increased to $6.71 \pm 1.62$ $\mathrm{mM}$, while when heavy exercise followed the moderate intensity warm-up lactate increased to only $5.08 \pm 0.99 \mathrm{mM}$ which was significantly less than the heavy warm-up. Thus, the intensity of the prior warm-up exercise had a significant impact on the lactate concentration immediately on starting the subsequent heavy exercise and it affects the final blood lactate concentration after the heavy exercise. Although this study did not have data for muscle lactate, previous investigations would suggest a strong correlation between muscle and blood concentrations (Linnarsson et al., 1974; Karlsson, 1971) so it seems safe to assume that muscle lactate could be elevated more during heavy exercise that followed a prior heavy warm-up than exercise that followed a moderate warm-up.

Overall, these results suggest that prior warm-up exercise is beneficial in accelerating the $\dot{Q}$ response to subsequent heavy exercise delivering more $\mathrm{O}_{2}$ to the working muscles so that $\dot{V} O_{2}$ can increase more rapidly as the metabolic machinery provides the substrates for oxidative phosphorylation. Moderate warm-up exercise improved $\dot{V} O_{2}$ and $\dot{Q}$ kinetics as much as prior heavy exercise but without the same elevation of $\dot{V} O_{2}, \dot{Q}$ and blood lactate.

\section{Application to elite distance running}

Elite distance running is never at a constant work rate. Frequently the early pace can be too rapid to be maintained and the middle part of the race involves a slightly reduced running speed for recovery. However, ultimate success is normally gained by the individual who maintains the greatest reserve for a well-timed sprint to the finish. There are several factors that contribute to athletic success including having a relatively high $\dot{V} O_{2 \max }$, excellent running economy and a high buffer capacity for the period of lactate production (Lucia et al., 2006; Denadai et al., 2006). In addition, this review has demonstrated the importance for the athlete to incur the smallest oxygen deficit possible by having rapid adaption of the $\mathrm{O}_{2}$ supply and utilization mechanisms as reflected in the kinetics of $\dot{V} \mathrm{O}_{2}$ and $\dot{Q}$. The fast kinetics for $\dot{V} \mathrm{O}_{2}$ will contribute to the maintenance of relatively high $\mathrm{PCr}$ reserves with low concentrations of lactate during the majority of the race. Thus, fast kinetics of $\dot{V} O_{2}$ and $\dot{Q}$ are essential and provide the greatest opportunity for athletic success.

\section{Acknowledgements}

Research support has been provided by the Natural Sciences and Engineering Research Council of Canada, and Egyptian Ministry of Higher Education and Scientific Research.

\section{References}

Brittain CJ, Rossiter HB, Kowalchuk JM, Whipp BJ (2001). Effect of prior metabolic rate on the kinetics of oxygen uptake during moderate-intensity exercise. Eur J Appl Physiol $86: 125-134$

Burnley M, Jones AM, Carter H, Doust JH (2000). Effects of prior heavy exercise on phase II pulmonary oxygen uptake kinetics during heavy exercise. J Appl Physiol 89:1387-1396

Denadai BS, Oritz MJ, Greco CC, de Mello MT (2006). Interval training at $95 \%$ and $100 \%$ of the velocity at $\dot{V} O_{2 \max }$ : effects on aerobic physiological indexes and running performance. Appl Physiol Nutr Metab 31:737-743

Faisal A, Beavers KR, Robertson AD, Hughson RL (2009). Prior moderate and heavy exercise accelerate oxygen uptake and cardiac output kinetics in endurance athletes. J Appl Physiol 106:1553-1563

Gerbino A, Ward SA, Whipp BJ (1996). Effects of prior exercise on pulmonary gas-exchange kinetics during high-intensity exercise in humans. J Appl Physiol 80:99-107 
Greenhaff PL, Campbell-O'Sullivan SP, Constantin-Teodosiu D, Poucher SM, Roberts PA, Timmons JA (2002). An acetyl group deficit limits mitochondrial ATP production at the onset of exercise. Biochem Soc Trans 30:275-280

Hohwu Christensen E Hogberg P (1950). Steady-state, $\mathrm{O}_{2}$-deficit and $\mathrm{O}_{2}$-debt at severe work. Arbeitsphysiologie 14:251-254

Hughson RL (2009). Oxygen uptake kinetics: historical perspective and future directions. Appl Physiol Nutr Metab 34:840-850

Hughson RL Morrissey MA (1982). Delayed kinetics of respiratory gas exchange in the transition from prior exercise. J Appl Physiol 52:921-929

Hughson RL, O'Leary DD, Betik AC, Hebestreit $H$ (2000). Kinetics of oxygen uptake at the onset of exercise near or above peak oxygen uptake. J Appl Physiol 88:1812-1819

Hughson RL, Tschakovsky ME, Houston ME (2001). Regulation of oxygen consumption at the onset of exercise. Exercise and Sports Science Reviews 29:129-133

Hughson RL Faisal A (2010). On the method of fitting cardiac output kinetics in severe exercise. Eur J Appl Physiol DOI 10.1007/s00421-0101787-x:

Karlsson J (1971). Lactate and phosphagen concentrations in working muscle of man with special reference to oxygen deficit at the onset of work. Acta Physiol Scand Suppl 358:7-72

Linnarsson D, Karlsson J, Fagraeus L, Saltin B (1974). Muscle metabolites and oxygen deficit with exercise in hypoxia and hyperoxia. J Appl Physiol 36:399-402

Lucia A, Esteve-Lano J, Olivan J, GomezGallego F, San Juan AF, Santiago C, Perez M, Foster C (2006). Physiological characteristics of the best Eritrean runners-exceptional running economy. Appl Physiol Nutr Metab 31:530-540

Ozyener F, Rossiter HB, Ward SA, Whipp BJ (2001). Influence of exercise intensity on the on- and off-transient kinetics of pulmonary oxygen uptake in humans. J Physiol (Lond) 533:891-902

Pringle JS, Doust JH, Carter H, Tolfrey K, Campbell IT, Jones AM (2003). Oxygen uptake kinetics during moderate, heavy and severe intensity 'submaximal' exercise in humans: the influence of fibre type and capillarisation. Eur J Appl Physiol 89:289-300

Rossiter HB, Ward SA, Doyle VL, Howe FA, Griffiths JR, Whipp BJ (1999). Inferences from pulmonary $\mathrm{O}_{2}$ uptake with respect to intramuscular [phosphocreatine] kinetics during moderate exercise in humans. J Physiol (Lond ) 518:921-932

Scheuermann BW, Hoetling BD, Noble ML, Barstow TJ (2001). The slow component of $\mathrm{O}_{2}$ uptake is not accompanied by changes in muscle EMG during repeated bouts of heavy exercise in humans. J Physiol (Lond ) 531:245-256

Tordi N, Perrey S, Harvey A, Hughson RL (2003). Oxygen uptake kinetics during two bouts of heavy cycling separated by fatiguing sprint exercise in humans. J Appl Physiol 94:533-541 